\title{
Infrared Properties of Cesium Bromide Prisms
}

\author{
Earle K. Plyler and Nicolo Acquista
}

\begin{abstract}
A prism of cesium bromide has been installed in a Perkins-Elmer spectrometer, and spectra have been observed in the region from 15 to $38 \mu$. This prism is of better optical quality than those of KRS-5, which makes it possible to obtain sharp images at the second slit of the spectrometer. Measurements have been made with this cesium bromide prism, which has a refracting angle of $55^{\circ}$, and the results have been compared with those obtained with a $26^{\circ}$ KRS-5 prism. The dispersion of cesium bromide from 15 to $38 \mu$ is about equal to KRS-5, but the effective resolution is about twice as great. A group of rotational lines of water vapor at $28.4 \mu$ is now clearly resolved into three components, the two narrow ones being separated by $1.8 \mathrm{~cm}^{-1}$. The long wavelength bands of carbon disulfide and chloroform have been measured in the vapor state, and it is possible, with the good definition available, to observe the $P, D$, and $R$ branches. In the spectral region beyond $33 \mu$, the stray radiation produces a considerable amount of the total deflection. The stray radiation was reduced to one-tenth of its value by the use of a roughened silver mirror located in the source box of the spectrometer.
\end{abstract}

\section{Introduction}

The extension of infrared measurements to wave. lengths greater than $25 \mu$ by the use of $\mathrm{KRS}-5$ prisms has been of considerable value in measuring the low lying fundamental bands of molecules. ${ }^{1}$ Although the KRS-5 prisms usually have poor optical quality, they have been used in many laboratories. The purpose of this investigation is to test another crystalline material, cesium bromide, for possible use in the infrared region between 25 and $40 \mu$. Previous measurements on crystals of this material grown at this Bureau showed that it was transparent to $40 \mu$ for a window $6 \mathrm{~mm}$ in thickness. ${ }^{2}$

In recent months $\mathrm{CsBr}$ crystals of good optical quality have been grown in industrial laboratories. Prisms obtained from two companies have been tested in a Perkin-Elmer spectrometer. One prism had a fault in the crystal which increased the stray radiation, but there was very little diffusion of the image on the slit. On comparing spectra obtained by this prism with those measured by the one without apparent flaws, no appreciable difference was noted in the results.

A cesium bromide prism with a refracting angle of $55^{\circ}$ was mounted in the Perkin-Elmer spectrometer, and it was placed at such an angle that the yellow lines of mercury could be brought on the exit slit when the drum of the spectrometer was set for large numbers. It was then possible to reach $40 \mu$ before the drum was turned the full way to the zero. The prism was adjusted in the spectrometer by the use of the visible lines of mercury.

1 Earle K. Plyler, J. Chem, Phys. 17, 218 (1949).

2 Earle K. Plyler and Francis P. Phelps, J. Opt. Soc. Am. 41, 209 (1951).
After the final adjustment of the instrument, some properties of the cesium bromide prism were determined. They are discussed in the following part of this paper.

\section{Results}

\subsection{Transmittance}

Transmittance of a cesium bromide prism with a $55^{\circ}$ refracting angle is sufficient to allow measurements to be made to $38 \mu$. This limit is about 1.8 microns smaller than the cut-off of a $26^{\circ} \mathrm{TlBr}-\mathrm{I}$ prism.

\subsection{Resolution and Definition}

The resolution of the cesium bromide prism is greater than that obtained with TlBr-I prisms. A practical test of resolution of the instrument, with a $\mathrm{CsBr}$ prism as the dispersing component, has been made. Figure 1 shows the resolution of the instrument in the region from 25 to $38 \mu$, the absorption bands being produced by water vapor in the air. Three closely spaced lines are resolved in the region of $28.4 \mu$, two of the lines resolved differing in wave number by only $1.8 \mathrm{~cm}^{-1}$. These lines could not be resolved with the $\mathrm{TlBr}$-I prism. The better resolution of the cesium bromide prism in the long wavelength region is made possible by its higher optical quality and its high dispersion, approximately equal to that of TlBr-I.

There is very little fuzziness or spreading out of the images of atomic spectral lines when they are focused on the second slit. This feature of the prism material makes it possible to follow rapid changes in absorption over small wavelength intervals. Many 
absorption bands of substances in the vapor state have $P, Q$, and $R$ branches. In measurements with the TlBr-I prism, it is not possible to observe band shapes on account of the poor definition of the image, but with the $\mathrm{CsBr}$ prism the band shapes can be observed for many molecules. An example of the observation of band shapes can be seen in figure 2 . The carbon disulfide band at $25.17 \mu$ is clearly resolved into $P, Q$, and $R$ branches. The $27.2 \mu$ band of chloroform, which appeared as a single maximum when measured by the TIBr-I prism, is now resolved into four components. The dotted part of the curve is an approximation of the true absorption; interference caused by water vapor partially masks the absorption due to the chloroform. The good definition of the prism is also advantageous for measuring spectra in that narrow absorption bands or lines can be measured to their true depths rather than as shallow diffuse bands.

\subsection{Dispersion}

The indices of refraction of cesium bromide have not been measured, but a good idea of the dispersion of the prism can be obtained by measuring the number of microns scanned for one revolution of the spectrometer drum. The prism was set for minimum deviation in the visible region and was off from the minimum deviation by a few degrees in the region from 24 to $37 \mu$. Figure 3 shows the number of microns included for one revolution of the drum. The results for eight measurements at intervals throughout the spectral range are well represented by the straight line in the figure. For comparison, a similar set of measurements for TlBr-I prism with a refracting angle of $26^{\circ}$ are also shown. Since the deviations of the two prisms are in the ratio of 2 to 1 , and the refracting angles are about in the same ratio, it appears that the dispersions of cesium bromide and $\mathrm{TlBr}-\mathrm{I}$ are comparable in the region from 24 to $38 \mu$.

\subsection{Stray Radiation}

The effect of stray radiation is large beyond $24 \mu$, and becomes a serious problem in measuring spectra with the cesium bromide prism. The transmittance of $\mathrm{TlBr}-\mathrm{I}$ is small in the near infrared region, and thus a filtering effect is produced; but there is no absorption by cesium bromide in the short wave.. length region and hence no filtering effect is produced. When the instrument is set for the long wavelength regions, the ratio of the stray radiation to the meas.. sured energy increases and is about 80 percent of the total energy at $37 \mu$. The amount of stray radiation present in an instrument is, in part, dependent on the accidental reflection from the interior of the spectrometer. Diaphragms of black paper were placed in various parts of the spectrometer, but with this shielding the stray radiation was reduced very little, which makes it appear that the stray radiation originates principally from the surfaces and interior of the prism. In order to cope with this situation, it is necessary to reduce the amount of the short wavelength energy which enters the spectrometer, since the stray radiation is primarily composed of the peak energy in the region from 1 to $5 \mu$ of the globar source.

Two types of filters, transmission and reflection, were tested for use in removing the stray radiation. It was found that satisfactory results were obtained by the use of a reflection filter which was made by roughening the surface of a polished silver mirror. The roughened silver mirror was placed in the source box of the spectrometer in place of the plane aluminum mirror. Figure 4 represents the percentage of the total energy contributed by the stray radiation at different wavelengths for the aluminum mirror, the rough and the very rough silver mirrors. The rough surface was produced by rubbing the polished mirror several times over 3/0-80 emery cloth, and the very rough mirror was produced by rubbing on 1/0-120 emery cloth. The very rough silver mirror reduces by a factor of ten the amount of stray radiation that is present with the aluminum mirror. The roughened mirrors do not reflect the long wavelength radiation quite as well as the polished mirrors, and it was found that the nonstray radiation was reduced to 85 percent of that obtained with the aluminum mirror. This reduction in effective energy was less than that found for transmission filters.

\section{Conclusion}

The higher optical homogeneity and the lower refractive index with approximately the same dispersion of the cesium bromide prism make it more suitable for measurements in the region from 24 to $38 \mu$ than a TIBr-I prism. Also, from about 20 to $24 \mu$, the results obtained with a cesium bromide prism are comparable with those obtained with a potassium bromide prism. For measurements covering the region from 2 to $38 \mu$, fair resolution can be obtained by the use of two prisms, sodium chloride and cesium bromide. However, for the highest resolution, prisms of lithium fluoride, calcium fluoride, and potassium bromide must be employed in the appropriate regions. The use of different prisms to cover the greatest span may be somewhat changed when cesium iodide becomes available. Small cyrstals of high transparency have been grown at this Bureau and an estimate of probable transparencies suggests that this material will make possible the extension of prism spectrometry to wavelengths greater than $50 \mu$. 


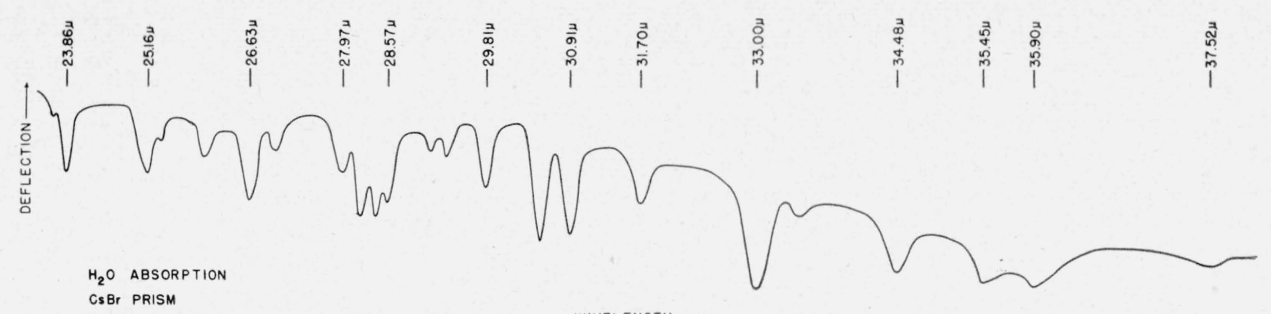

WAVELENGTH $\longrightarrow$

Figure 1. Infrared spectrum of water vapor from 23.6 to $3 \% .5 \mu$.

$\mathrm{H}_{2} \mathrm{O}$ absorption $\mathrm{CsBr}$ prism.

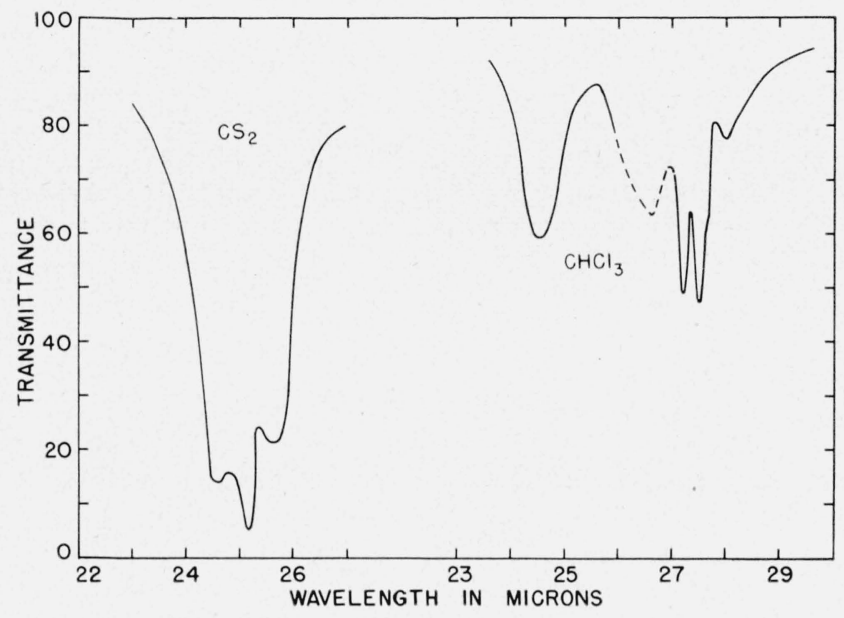

FIGURE 2. Absorption bands of carbon disulfide at $25.2 \mu$ and those of chloroform at $28 \mu$.

$\mathrm{CS}_{2}$ cell length, $10 \mathrm{~cm}$; pressure, $19 \mathrm{~cm}$; $\mathrm{CHCl}_{3}$ cell length, $160 \mathrm{~cm}$; saturated pressure.

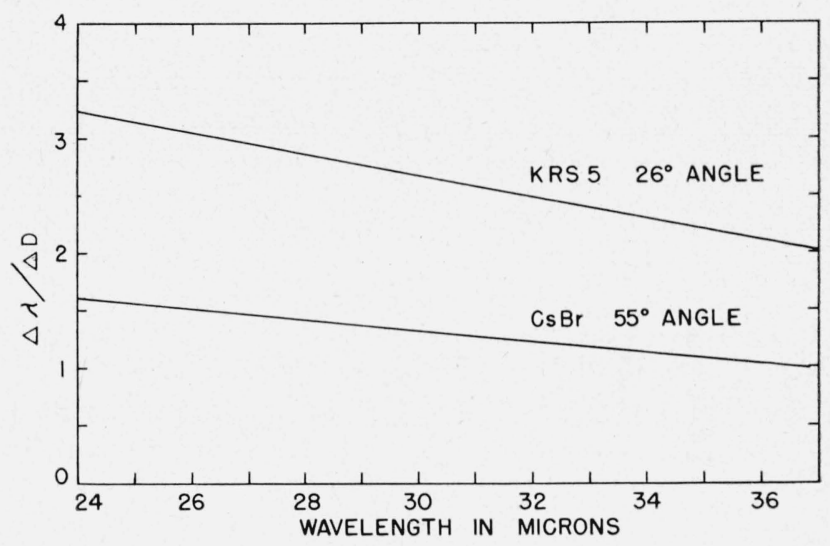

FIguRE 3. Change in wayelength per revolution of spectrometer drum for prisms of KRS-5 and cesium bromide in the region from 24 to $37 \mu$.

K RS- $5,26^{\circ}$ angle; $\mathrm{CsBr}, 55^{\circ}$ angle.

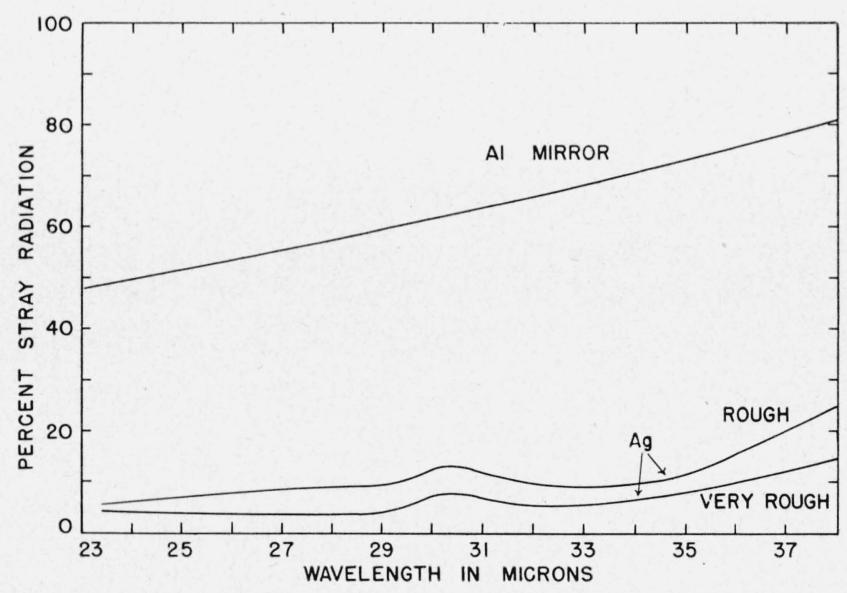

Figure 4. Percentage of stray radiation in the long-wavelength region for different reflectors.

Washington, April 8, 1952. 